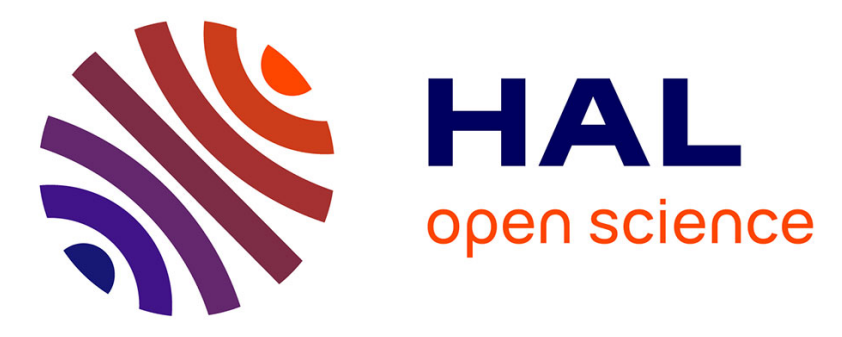

\title{
Structural Transitions in MIL-53 (Cr): View from Outside and Inside
}

Alexander Neimark, François-Xavier Coudert, Carles Triguero, Anne Boutin, Alain Fuchs, Isabelle Beurroies, Renaud Denoyel

\section{To cite this version:}

Alexander Neimark, François-Xavier Coudert, Carles Triguero, Anne Boutin, Alain Fuchs, et al.. Structural Transitions in MIL-53 (Cr): View from Outside and Inside. Langmuir, 2011, 27 (8), pp.4734-4741. 10.1021/la200094x . hal-02113309

\section{HAL Id: hal-02113309 \\ https://hal.science/hal-02113309}

Submitted on 28 Apr 2019

HAL is a multi-disciplinary open access archive for the deposit and dissemination of scientific research documents, whether they are published or not. The documents may come from teaching and research institutions in France or abroad, or from public or private research centers.
L'archive ouverte pluridisciplinaire HAL, est destinée au dépôt et à la diffusion de documents scientifiques de niveau recherche, publiés ou non, émanant des établissements d'enseignement et de recherche français ou étrangers, des laboratoires publics ou privés. 


\title{
Structural Transitions in MIL-53 (Cr): View from Outside and Inside
}

Alexander V. Neimark, ${ }^{*},{ }^{, a b}$ François-Xavier Coudert, ${ }^{* a}$ Carles Triguero, ${ }^{a}$ Anne Boutin, ${ }^{\mathrm{c}}$

Alain H. Fuchs, ${ }^{\mathrm{a}}$ Isabelle Beurroies, ${ }^{\mathrm{d}}$ and Renaud Denoyel ${ }^{\mathrm{d}}$

a - Chimie ParisTech (École Nationale Supérieure de Chimie de Paris), CNRS and Univ. Pierre et Marie Curie, Paris, France. b - Department of Chemical and Biochemical Engineering, Rutgers University, New Jersey, USA. c - Chemistry Department, École Normale Supérieure, CNRS, UPMC, Paris, France; $\mathrm{d}$ - Laboratoire Chimie Provence, CNRS and Universités d'AixMarseille, Marseille, France.

* corresponding authors, emails: aneimark@rutgers.edu; fx.coudert@chimie-paristech.fr

\begin{abstract}
We present a unified thermodynamic description of the breathing transitions between large pore (lp) and narrow pore (np) phases of MIL-53 (Cr) observed during the adsorption of guest molecules and the mechanical compression in the process of mercury porosimetry. By revisiting recent experimental data on mercury intrusion and in-situ XRD during $\mathrm{CO}_{2}$ adsorption, we demonstrate that the magnitude of the adsorption stress exerted inside the pores by guest molecules, which is required for inducing the breathing transition, corresponds to the magnitude of the external pressure applied from the outside that causes the respective transformation between Ip and np phases. We show that, when a stimulus is applied to breathing MOFs of MIL53 type, these materials exhibit small reversible elastic deformations of $\mathbf{l p}$ and $\mathbf{n p}$ phases of the order of $2-4 \%$, while the breathing transition is associated with irreversible plastic deformation that leads to up to $\sim 40 \%$ change of the sample volume and a pronounced hysteresis. These results shed light on the specifics of the structural transformations in MIL-53 (Cr) and other soft porous crystals (SPC).
\end{abstract}


The structural transitions in metal-organic frameworks (MOF) known as breathing have been attracting a lot of attention from both the material scientists, who discovered and explored potential practical applications of this intriguing phenomenon, and the theoreticians, who attempted to establish the thermodynamics foundations and simulation models to understand its physico-chemical and mechanical mechanisms. ${ }^{1,2,3,4,5}$ The most prominent breathing transitions were observed in the process of gas adsorption in some MOFs of the MIL-53 family. The MIL53 framework is made of parallel one-dimensional $\mathrm{M}(\mathrm{OH})$ chains $\left(\mathrm{M}=\mathrm{Al}^{3+}, \mathrm{Cr}^{3+}\right)$, linked together by 1,4-benzenedicarboxylate (BDC) ligands to form linear diamond-shaped channels that are wide enough to accommodate small guest molecules. ${ }^{6,7,8}$ This structure may oscillate (or "breathe") between two distinct conformations called a large-pore phase (lp) and a narrow-pore phase (np; see Figure S1), which have a remarkable difference in cell volume of up to $40 \%$. The conditions, at which the breathing of MIL-53 happens, have been widely studied earlier, and it was shown in particular to be triggered by changes in temperature, ${ }^{9}$ as well as adsorption of some gases and fluid mixtures at room temperature, but not others. ${ }^{10,11,12,13,14}$ Recent work has been successful in rationalizing the conditions for the occurrence of breathing in MIL-53, ${ }^{15}$ by considering the issue from the point of view of thermodynamics in the osmotic ensemble, which is the statistico-mechanical ensemble adapted for the study of adsorption in a flexible host. ${ }^{16}$ This thermodynamic analysis was then extended to fluid mixtures and to an investigation of temperature dependence, which allowed to predict complete pressure-composition and pressuretemperature phase diagrams for various adsorbates in MIL-53. ${ }^{17,18,19}$

Recently, the authors ${ }^{20}$ suggested a rationale for breathing transitions driven by guest molecules adsorbed inside the pores. It was shown that the adsorbed molecules induce a significant stress inside the elastic framework that can be of the order of several to tens of MPa. 
This adsorption stress, $\sigma_{a}$, may be either positive or negative depending on the loading and, thus, it may induce either framework expansion or contraction. The breathing transitions from Ip to $\mathbf{n p}$ phase and back were treated as spontaneous non-equilibrium transitions occurring upon developing a certain threshold stress $\sigma^{*}$, which the respective phase cannot withhold. Based on this adsorption stress model and the threshold stress ansatz, the authors described on a semiquantitative level the breathing transitions driven by $\mathrm{Xe}$ and $\mathrm{CH}_{4}$ adsorption on MIL-53 (Al). ${ }^{20,21}$

In a recent publication, Beurroies et $\mathrm{al}^{22}$ demonstrated that the structural transitions between $\mathbf{l p}$ and $\mathbf{n p}$ phases can be induced by uniform mechanical compression of the sample from outside in the process known as mercury porosimetry. ${ }^{23}$ The powder of MIL-53 (Cr) particles was immersed into liquid mercury subjected to the external pressure. Upon the increase of pressure, mercury, being a non-wetting fluid, was forced to penetrate into interparticle voids of micrometer size, but it was not intruded into the internal pores of nanometer size, thus exerting a uniform pressure on the particles. The Ip-np transition in the process of mercury intrusion and the reverse np-lp transition in the process of mercury extrusion occurred at significantly different pressures, giving rise to a pronounced hysteresis loop formed by intrusionextrusion curves.

In this work, we extended the thermodynamic approach based on the osmotic ensemble ${ }^{16}$ coupled with the adsorption stress model ${ }^{20}$ suggested for the description of adsorption-induced breathing transitions, to describe mechanically-driven transitions observed by Beurroies et al. with MIL-53 (Cr). ${ }^{22}$ To this end, we revisited the mercury intrusion data and compared it with $\mathrm{CO}_{2}$ adsorption and in situ XRD data collected on MIL-53 (Cr) by Serre et al. ${ }^{10}$ We demonstrate that the magnitude of the adsorption stress applied inside the pores by adsorbed molecules, which is required for inducing the breathing transition, corresponds to the magnitude of the 
external pressure applied from the outside that causes irreversible plastic transformation between lp and np phases. This conclusion allows one to predict the adsorption-induced phase transformations of MOF based on the mechanical contraction experiments.

\section{View from outside: What can one learn from the mercury intrusion-extrusion} experiments?

In the mechanical compression experiments, a powder of MIL-53 (Cr) particles is placed in the sample cell of the mercury porosimeter, which represents a piston-cylinder assembly. To ensure that the particles are in the Ip state the sample was initially immersed in methanol which is known to open the MOF structure. Then it was outgassed at $150^{\circ} \mathrm{C}$ to remove adsorbed species, cooled and transferred as quickly as possible in the mercury porosimeter cell for analysis. In the mercury apparatus, a primary vacuum is set lower than $5 \mathrm{~Pa}$. Then, a given amount of mercury is added into the sample cell on the top of the powder and the mercury pressure is gradually increased by a hydraulic pump. The cell volume underneath the piston is measured as well as the equilibrium external pressure applied through the piston to the mercury and thus transmitted to the powder particles. The results of the mercury intrusion-extrusion cycles are presented in Figure 1. Note that the raw data ${ }^{22}$ was corrected for the mercury compressibility and the pressure transducers hysteresis using blank calibration experiments on mercury intrusion-extrusion inside the empty sample cell. This correction is necessary for quantitative analyses of the experimental data at high pressures, especially in the region of hysteresis. The graph in Figure 1 gives the volume of mercury $V$ penetrated at the given external pressure $P$ into the volume occupied by the powder; the external pressure in the initial state is null, $P=0$. The consecutive stages of the intrusion-extrusion process are shown by different colors: the blue curve depicts the primary intrusion obtained by raising $P$ from 0 to $410 \mathrm{MPa}$, the 
red curve depicts the primary extrusion obtained by reverting the process at $P=410 \mathrm{MPa}$ and reducing $P$ to $0.34 \mathrm{MPa}$, the violet curve depicts the secondary intrusion obtained by reverting the process at $P=0.34 \mathrm{MPa}$ and raising $P$ again to $410 \mathrm{MPa}$, and finally the orange curve depicts the secondary extrusion obtained within the same pressure limits as the primary one. The almost ideal coincidence of the primary and secondary extrusion curves and co-linearity of the primary and secondary intrusion curves at $P>10 \mathrm{MPa}$ show the reliability of the presented experiment.

The mercury porosimetry technique is a standard tool for pore size characterization of porous solids. ${ }^{23}$ It employs the fact that being a non-wetting fluid, mercury penetrates into the pores only when the external pressure exceeds the capillary pressure determined by the LaplaceWashburn equation, $P=4 \gamma \cos \theta / D$, where $D$ is the effective pore diameter, $\gamma$ is the mercury surface tension $(\gamma=0.485 \mathrm{~N} / \mathrm{m})$, and $\theta$ in the contact angle, which for most solids varies from $130^{\circ}$ to $145^{\circ}[14]$. Thus, in order to intrude mercury into micrometer-wide pores, the external pressure should exceed $0.2 \mathrm{MPa}$, and, respectively, filling of micropores $(D<2 \mathrm{~nm})$ may occur only at an external pressure exceeding $\sim 600 \mathrm{MPa}$. These simple estimates help understand the different steps in the mercury intrusion-extrusion cycle. The first step on the primary intrusion curve at low pressures $P<0.2 \mathrm{MPa}$ (Figure 1, point A) characterizes the powder compaction into a dense random packing without mercury penetration into the gaps between micrometer size particles of MIL-53 crystals. The second step, at $0.2 \mathrm{MPa}<P<10 \mathrm{MPa}$ (from point A to point B), reflects mercury filling of the interparticle voids in the dense packing of particles without penetration into intraparticle pores. Indeed, the pores in MIL-53 are in the micropore range $(<2 \mathrm{~nm})$ and cannot be filled at pressures smaller than $600 \mathrm{MPa}$ that exceeds the maximum pressure of $410 \mathrm{MPa}$ achieved in given experiments. 
1.1 Particle size analysis. From the range of pressures, $0.2-10 \mathrm{MPa}$, corresponding to the interparticle filling, one can estimate the particle size distribution using the conventional method of Mayer and Stowe. ${ }^{24}$ The calculated differential particle size distribution is presented in Figure 2 as the mass fraction of particles of given diameter. In the basic Mayer-Stowe equation, $P=\kappa \gamma / D_{\mathrm{p}}$, which relates the intrusion pressure $P$ and the particle diameter $D_{\mathrm{p}}$, the mercury surface tension $\gamma$ was taken as $0.485 \mathrm{~N} / \mathrm{m}$, and the dimensionless Mayer-Stowe constant $\kappa$, that typically varies in the range of 6-13, was taken as $10 .^{25}$ The average particle diameter is $D_{\mathrm{p}}=$ $2.7 \mu \mathrm{m}$, and the width of the distribution is $\sim 0.8 \mu \mathrm{m}$. This is in agreement with the results of Khan and Jhung, where scanning electron microscopy (SEM) images showed relatively homogeneous particles of size $2-3 \mu \mathrm{m}$, though on a different batch of MIL-53 particles. $^{26}$

1.2 Elastic deformation and bulk modulus estimate. Neglecting mesopores, which may exist in small amounts due to possible defects and imperfections of MIL-53 crystals, we assume that mercury does not penetrate the intraparticle pores in the whole range of external pressures in given experiment. Upon the filling of interparticle pores, mercury exerts the uniform hydrostatic pressure on the particles and the further gradual increase of the intruded volume at $10 \mathrm{MPa}<P<$ $35 \mathrm{MPa}$ reflects the elastic compression of particles, which are in the lp phase. This elastic compression should be reversible. From the slope of the intrusion curve in the region of linear elastic deformation, one can determine the volumetric elastic modulus (or bulk modulus) $K_{\mathrm{lp}}$ of the Ip phase of MIL-53 crystals, as $K=V_{0}(\partial P / \partial V)$ with $V_{0}$ being the reference volume of the respected phase. We have calculated the bulk modulus of the Ip phase from the second mercury intrusion curve, for which the linear regime is clear (see Figure S2). The value obtained is $K_{\mathrm{lp}} \sim 2 \mathrm{GPa}$, which is in line with the values of bulk modulus reported in the literature for other MOF structures, which typically fall in the range $1.5-12 \mathrm{GPa}^{27,28}$ (It is worth noting that 
HKUST-1 was reported to have a much higher bulk modulus than other members of the MOF family, at $\sim 30 \mathrm{GPa}^{29}$ ) Similarly, the bulk modulus of the np phase can be estimated from the primary and secondary extrusion curves, which overlap reasonably in the region of elastic deformation at $P>30 \mathrm{MPa}$. Thus found value of $K_{\mathrm{np}} \sim 10 \mathrm{GPa}$ is larger than that of the Ip phase. Indeed, the np phase, which is denser than the Ip phase, is also less compressible. We can also estimate the elastic domain of deformation, i.e. the maximal variation of the crystal volume observed within the region of elastic deformation. The unit cell volume variation in the elastic regime for both phases is small (few percents), as indicated in Table 1.

1.3. Structural lp-np and np-lp transitions. The region of elastic contraction terminates with a prominent step at $\mathrm{P}^{*} \sim 55 \mathrm{MPa}$, which was treated as the transition from the lp to the $\mathbf{n p}$ phase. This transition is highlighted on Fig. 1 with a vertical arrow. The volume of intruded mercury during this step, of $394 \AA^{3} /$ u.c., agrees within $\sim 10 \%$ with the difference in the volumes of Ip and $\mathbf{n p}$ phases as obtained from crystallographic data, namely $440 \AA^{3} /$ u.c. The difference between these two values, of the order of $10 \%$, may be ascribed to the continued existence of a fraction of Ip phase in the experimental sample, as was observed upon $\mathrm{Xe}$ and $\mathrm{CO}_{2}$ adsorption. ${ }^{21,30}$ According to the ansatz that the $\mathbf{l p - n p ~ t r a n s i t i o n ~ o c c u r s ~ u p o n ~ a c h i e v i n g ~ i n ~ t h e ~} \mathbf{l p}$ phase a certain threshold negative stress $\sigma^{*}$, the value of the external pressure $P^{*}{ }_{\text {lp }}$ of the lp-np transition gives the estimate of the maximum negative stress $\sigma^{*}$, which the lp phase can withhold, namely $P^{*}{ }_{\mathbf{l p}}=-\left(\sigma^{*} \mathbf{l p}-\sigma_{\mathbf{l p}}^{0}\right)$, where $\sigma_{\mathbf{l p}}^{0}$ is the pre-stress inherent to the dry material in the absence of mechanical constraint at $P=0$. Moreover, the authors hypothesized that the threshold stress $\sigma^{*}$ depends on the particle size, the smaller the particle the smaller the threshold stress. ${ }^{20}$ Following this hypothesis, we can associate the width of the lp-np transition step $P^{*}{ }_{\mathbf{p}}=$ 55 ( \pm 15$) \mathrm{MPa}$ with the distribution of the threshold stress due to the particle size distribution. 
Thus, using the particle size distribution determined from the interparticle penetration region, one can correlate the threshold pressure of the lp-np transition and the particle size.

Upon further increase of pressure the intruded volume increases linearly and reflects the elastic compression of particles, which are now in the np phase. The elastic compression is reversible as seen from the extrusion curve, which coincide with the intrusion one within experimental errors. From the slope of the intrusion-extrusion curve in this region, one can determine the volumetric elastic modulus of the $\mathbf{n p}$ phase. The elastic expansion of the $\mathbf{n p}$ phase in the process of mercury extrusion terminates with a stepwise np-lp transition, which occurs in the range of pressures $0<P<15 \mathrm{MPa}$. Similarly to the lp-np transition, according to the threshold stress ansatz, the np-lp transition occurs upon achieving a certain threshold (now positive) stress, $P^{*}{ }_{\mathbf{n p}}=10( \pm 10) \mathrm{MPa}$, which may depend on the particle size. The np-lp transition is, again, highlighted on Fig. 1 with a vertical arrow.

Note that the external pressure in the intrusion experiment equals the negative stress calculated from the pre-stress in the reference evacuated sample at $P=0$. For the lp phase, the situation is straightforward, since this reference state is the starting point of the intrusion experiment. However, the np phase of the MIL-53 (Cr) structure is unstable at the temperature of these experiments (around $300 \mathrm{~K}$ ), so that no such reference state exist. To remedy the situation, we can considered a hypothetical np state, which would be obtained by extrapolation of the linear elastic expansion regime down to $P=0$, as the reference state for thermodynamic calculations. This extrapolation is shown in Figure 1 by a dotted line (lower panel; the reference state is marked as point $C$ ).

\section{View from inside: adsorption-driven structural transitions.}


Gas adsorption was the first physical stimulus, for which breathing transitions in MIL-53 were observed, well before it was demonstrated that temperature and mechanical pressure could also trigger the lp-np structural transition. Adsorption of many gases inside MIL-53 (Al, Cr) is characterized by step-wise isotherms indicative of breathing transitions between $\mathbf{n p}$ and $\mathbf{l p}$ phases, which occur at certain gas pressures depending on the nature of the guest molecules and the temperature. The criteria for observing the breathing phenomenon in terms of MIL-53 properties and fluid/solid interactions have been defined from the thermodynamic considerations. It was shown that breathing is a general phenomenon that occurs within a certain temperature range for all guest molecules. ${ }^{19,21}$ Noteworthy, in the case of MIL-53 adsorbents, the two consecutive breathing transitions take place, $\mathbf{l p}-\mathbf{n p}$ at low gas pressure and $\mathbf{n p}-\mathbf{l p}$ at high gas pressure. The reverse $\mathbf{l p - n p}$ and $\mathbf{n p - l p}$ transitions occur in the process of desorption in a hysteretic manner. Analyses of adsorption isotherms provide the quantitative information about the breathing transitions in terms of the difference of adsorption capacities of $\mathbf{n p}$ and $\mathbf{l p}$ phases and, combined with in situ XRD patterns, the difference of unit cell geometries.

In the pioneering paper on $\mathrm{CO}_{2}$ adsorption in MIL-53 (Cr), Serre et al. reported in situ Xray diffraction experiments performed during adsorption/desorption cycles at room temperature. ${ }^{10}$ Revisiting this data on the adsorption-driven breathing obtained on the same structure as the mercury porosimetry experiments allows us to compare quantitatively the adsorption-driven breathing to that driven by mechanical pressure.

2.1. Analysis of in-situ XRD patterns. The isotherms and pressure-dependent XRD patterns from Ref. 10 are reproduced in Figure 3, for one adsorption/desorption cycle. The step seen on the adsorption isotherm can be attributed to the $\mathbf{n p - l p}$ transition, i.e. the second, high pressure breathing transition upon adsorption. The first, low-pressure lp-np transition happening 
around $0.3 \mathrm{bar}^{31}$ is not seen here. The evolution of the X-ray diffraction pattern upon adsorption and desorption shows a clear phase transition between the np and lp structures, respectively characterized by the peaks at $2 \theta$ around $5^{\circ}$ and $3.9^{\circ}$. The transition can clearly be seen as a jump from one structure to another, with no intermediate XRD peak being observed. Moreover, it can be seen that, within a narrow range of pressure around the transition, the two phases can coexist in the experimental sample, as the two characteristic XRD peaks are observed simultaneously. Note that this does not mean necessarily the phase coexistence within one MIL-53 (Cr) crystal; in a powder sample, some crystals can be in the lp phase and the others in the np phase.

In addition, we also see that the position of the XRD peaks of each structure shifts slightly during adsorption, which is an indication of the elastic deformation of each phase. This deformation is due to the adsorption stress created by the presence of $\mathrm{CO}_{2}$ in the pores of the material. By using a simple geometric model of the main feature of MIL-53 flexibility, we were able to map these small displacements of the XRD peaks onto variations of unit cell volume. The details of this model (and equations) are given in Supplementary Information.

The unit cell volume changes thus calculated are reported for the Ip phase in Figure 4 (the plot for the np phase is less instructive as the amplitude of variations is much smaller). The relative variation of volume upon adsorption, of around $1.5 \%$, is comparable to that measured from the mercury intrusion experimental data, indicating a good agreement between the two completely different sets of experimental results (and validating the geometric model used to process the XRD peak positions). Moreover, it can be noted that, because the measurements used for Figure 4 correspond to the high-pressure range of the adsorption and desorption isotherms, we are in a region of positive and increasing adsorption stress: as $\mathrm{CO}_{2}$ pressure increases, more molecules are packed into the nanopores of MIL-53, leading to an outward stress. These 
experimental measurement are thus in agreement with the picture provided by the stress model proposed in our earlier work. $^{20}$

2.2. Analysis of the adsorption isotherms. We have further applied the stress model ${ }^{20}$ to the adsorption data presented in Figure 3 left, combining the information we can extract from the adsorption isotherms with the data extracted from the mercury intrusion experiments, such as the bulk moduli of the Ip and np phases and the values of threshold stresses corresponding the breathing transitions. All this information leads to a unique set of the parameters for the stress model of MIL-53 breathing, which are given in Table 1. It is noteworthy that, while we have previously used the stress model to provide for a qualitative understanding of the $\mathrm{Xe}$ - and $\mathrm{CH}_{4}$ driven breathing of MIL-53 (Al), introducing information from mechanical experiments has now enabled us to calculate quantitatively the actual adsorption stress in the material.

\section{Unified description of sample deformation engendered by external pressure and}

\section{adsorption inside the pores.}

The equilibrium elastic deformation of a porous solid immersed in a fluid (gas or liquid) thermostated reservoir, which may contain one adsorbing species, is determined from the following fundamental equation,

$$
\begin{aligned}
& d \Omega\left(\mu, p_{e x t}\right)=d\left[F_{s}^{0}+\frac{1}{2} K_{0} V_{0}^{-1}\left(V-V_{0}\right)^{2}+p_{e x t} V+\Omega_{a}(\mu, V)\right]=N d u+V d p_{e x t} \\
& \Omega\left(\mu, p_{e x t}\right)=F_{s}^{0}+\frac{1}{2} K_{0} V_{0}^{-1}\left(V-V_{0}\right)^{2}+p_{e x t} V+\Omega_{a}(\mu, V)
\end{aligned}
$$


where $\Omega\left(\mu, p_{\text {ext }}\right)$ is the osmotic ensemble thermodynamic potential of the combined solid + fluid system at the given fluid pressure $p_{\text {ext }}$ and chemical potential $\mu$ of the adsorbate, $V$ is the current sample volume, $V_{0}$ is the sample volume in the evacuated (or "dry") state at $p_{\text {ext }} \rightarrow 0$ and $\mu \rightarrow-\infty$, which is considered as the reference state for thermodynamic analysis, $F_{s}^{0}$ is the solid free energy in the dry state, $K_{0}$ is the bulk modulus (or volumetric elastic constant) of the solid in the dry state, and $\Omega_{a}(\mu, V)$ is the grand thermodynamic potential of the adsorbate at given $\mu$ and $V$. Eq. 1 implies that the changes of the solid free energy with respect to the dry state are accounted by a harmonic elastic potential $\frac{1}{2} K_{0} V_{0}^{-1}\left(V-V_{0}\right)^{2}$. In this simplest consideration, we ignore a possible anisotropy of the solid and characterize the elastic deformation of the framework by the volumetric strain

$$
\varepsilon=\frac{\Delta V}{V_{0}}=\frac{V-V_{0}}{V_{0}}
$$

The threshold stress ansatz implies that the regions of elastic deformations are limited by the minimum negative stress $\sigma^{*}$ lp and respectively minimum negative strain $\varepsilon^{*}{ }_{1 p}$ in the case of $\mathbf{l p}$ phase and the maximum positive stress $\sigma^{*}{ }_{n p}$ and respectively maximum positive strain $\varepsilon^{*}{ }_{n p}$ in the case of $\mathbf{n p}$ phase. Ignoring the necessary deviations from the linear elasticity in the vicinity of threshold points, the strain-stress dependencies for the $\mathbf{l p}$ and $\mathbf{n p}$ phases are presented by two straight lines terminated at the threshold points, Figure 5. The slopes of these lines correspond to the respective bulk modulus $K_{\mathrm{Ip} / \mathbf{n p}}$ of the $\mathbf{l p}$ and $\mathbf{n p}$ phases. The mechanically driven transitions in the process of mercury intrusion-extrusion occurring upon achieving the threshold stress are depicted by the vertical lines in Fig.5. The resulting hysteresis loop is a mirror image of the experimental mercury intrusion-extrusion cycle in Figure 1 formed by the elastic deformation 
curves bounded by the vertical lines corresponding to the lp-np and np-lp transitions in the crystals of the mean size.

The equilibrium deformation is determined by minimization of the thermodynamic potential $\Omega\left(\mu, p_{\text {ext }}\right)$ with respect to the sample volume $V$ at given $p_{\text {ext }}$ and $\mu$ that brings about a general relation between the volumetric strain $\varepsilon$ and stress $\sigma$ in the form of the Hooke law,

$$
\sigma=K_{0} \varepsilon=\sigma_{a}-p_{e x t}
$$

Here, we introduced the adsorption stress ${ }^{32,33}$,

$$
\sigma_{a}\left(V_{0}\right)=-\left.\left(\frac{\partial \Omega_{a}}{\partial V}\right)\right|_{\mu, V=V_{0}}
$$

as the derivative from the grand thermodynamic potential with respect to the sample volume $V$ at given external conditions of constant $\mu$ and $p_{\text {ext }}$. Note that for one component fluid $p_{\text {ext }}$ and $\mu$ are related through the equation of state; that is in adsorption experiments, $p_{\text {ext }}$ equals the gas pressure $p$, and in the mercury intrusion experiments $p_{\text {ext }}$ equals the applied pressure $P$.

Eq. 3 constitutes the basis for a unified treatment of the mechanical and adsorption-driven deformation of MOFs. Indeed, the sample deformation in the process of adsorption should be similar to that in the process of mechanical compression provided the equality of the stress. We may conclude that assuming that both the adsorption experiment and compression experiment begin from the same initial dry evacuated state, the same sample deformation during adsorption and compression is achieved, when

$$
P=-\sigma_{a}(\mu)+p
$$


Eq. 5 allows us to predict the adsorption driven elastic deformation based of compression experiments, from which the stress-strain dependence (Fig. 5) can be derived, and vice versa. This conclusion implies that the adsorption stress $\sigma_{a}(\mu)$ can be calculated independently, for example, by employing an empirical Langmuir adsorption equation as it was done in our previous works for $\mathrm{Xe}$ and $\mathrm{CH}_{4}$ adsorption on MIL-53 (Al). ${ }^{20,21}$

The variation of the cell volume of the MIL-53 $(\mathrm{Cr})$ crystal in the process of $\mathrm{CO}_{2}$ adsorption is a function of the adsorption stress that can be calculated based on the stress model. ${ }^{20}$ Following our earlier treatment of Xe sorption in MIL-53 (Al), we approximated the adsorption isotherms on the $\mathbf{l p}$ and $\mathbf{n p}$ crystals with the Langmuir adsorption equation, $N(p)=N_{0} K_{\mathrm{H}} p /\left(N_{0}+K_{\mathrm{H}} p\right)$, with the unit cell capacity $N_{0}$ and the Henry constant $K_{\mathrm{H}}$ determined from the fitting of the experimental data. ${ }^{10}$ The adsorption stress $\sigma_{a}(\mu)$ was calculated according to Eq. 4, which in the case of Langmuir adsorption isotherm is directly related to the gas pressure, ${ }^{20}$

$$
\sigma_{s}(p)=R T\left\{\frac{\mathrm{d} N_{0}}{\mathrm{~d} V_{c}}\left[\ln \left(1+K_{\mathrm{H}} p / N_{0}\right)-\left(\frac{K_{\mathrm{H}} p / N_{0}}{1+K_{\mathrm{H}} p / N_{0}}\right)\right]+\frac{\mathrm{d} K_{\mathrm{H}}}{\mathrm{d} V_{c}}\left(\frac{p}{1+K_{\mathrm{H}} p / N_{0}}\right)\right\}
$$

In addition to $N_{0}$ and $K_{\mathrm{H}}$, the adsorption stress (6) depends on the variations of these quantities with respect to the cell volume, $\mathrm{d} N_{0} / \mathrm{d} V_{c}$ and $\mathrm{d} K_{\mathrm{H}} / \mathrm{d} V_{c}$. It is noteworthy, that while $\mathrm{d} N_{0} / \mathrm{d} V_{c}$ is apparently positive, $\mathrm{d} K_{\mathrm{H}} / \mathrm{d} V_{c}$ is in general negative. This gives rise to a nonmonotonic variation of the adsorption stress in the course of adsorption, as shown in ref. 20. In the Henry region at low pressures, $\sigma_{s}(p) \approx R T p \mathrm{~d} K_{\mathrm{H}} / \mathrm{d} V_{c}$ is negative and decreasing, that corresponds to the sample contraction. In the saturation region at high pressures, $\sigma_{s}(p) \approx R T\left[\mathrm{~d} N_{0} / \mathrm{d} V_{c} \ln \left(K_{\mathrm{H}} p / N_{0}\right)+\mathrm{d} K_{\mathrm{H}} / \mathrm{d} V_{c}\left(N_{0} / K_{\mathrm{H}}\right)\right]$ increases and may even become 
positive, leading to the sample expansion. Based on the threshold stress ansatz, the values of $\mathrm{d} N_{0} / \mathrm{d} V_{c}$ and $\mathrm{d} K_{\mathrm{H}} / \mathrm{d} V_{c}$ were determined from Eq. 5 equating the characteristic mercury pressures of lp-np and $\mathbf{n p - l p}$ transitions observed in the porosimetry experiments (vertical lines at the threshold pressures in Figure 5) and the adsorption stress (Eq. 6) of the respective transitions in the adsorption experiments (Figure 3, left). The parameters on the $\mathrm{CO}_{2}$ adsorption isotherms on $\mathbf{l p}$ and $\mathbf{n p}$ phases are given in Table 2.

Noteworthy, the system scans the different regions on the stress-strain diagram (Figure 5) during the adsorption-desorption cycle and during the intrusion-extrusion cycle. Indeed, in the compression experiments the stress exerted by the external pressure is always negative, while the adsorption stress may be positive due to the packing effects at high loading. At the same time, the negative adsorption stress is limited in the adsorption experiments due to the competition between the guest-host attractive interactions and the repulsive guest-guest interactions. However, the most important conclusion is that in the process of external compression and in the process of adsorption, the host framework experiences the same strain-stress elastic behavior and the lp-np transitions occur upon achieving the same threshold stress, which can be either directly measured in the mechanical experiments or calculated from the adsorption isotherms.

\section{Conclusions: how soft are Soft Porous Crystals?}

In the recent literature, flexible MOFs which exhibit adsorption-driven elastic deformation, gating, and breathing phenomena, of which MIL-53 is one of the most characteristic examples, were named soft porous crystals (SPC). ${ }^{5}$ Our study shows that softness of SPCs can be very different. Namely, one should distinguish between the structures like the swelling MIL- $88^{34}$, or 
interpenetrated dynamic frameworks, ${ }^{35}$ which change their volume gradually with respect to the applied stimulus of increasing magnitude, and the structures like breathing MOFs of MIL-53 type considered here. These latter structures feature regions of small reversible elastic deformations within relatively wide ranges of variation of the stimulus terminated by large irreversible plastic deformations occurring in a stepwise manner, which lead to hysteretic phase transformations.

The mechanical compression experiments with mercury porosimeters provide not only qualitative but also valuable quantitative information about the mechanical properties of Ip and np phases and the breathing transitions, including elastic modules of respective phases, the extent of the elastic deformation, and threshold stresses leading to the phase transformations, as well as the particle size distribution and correlations between the threshold stress and the particle size. The quantitative estimates extracted from the mercury porosimetry data on MIL-53 (Cr) sample correlate with the results of interpretation of the independent data on $\mathrm{CO}_{2}$ adsorption isotherms and in situ XRD patterns collected in the process of $\mathrm{CO}_{2}$ adsorption. The combined body of data from adsorption and mechanical experiments provides a solid foundation for a reliable theoretical consideration of the breathing transitions in SPCs.

The osmotic ensemble thermodynamic analysis based on the assumption of the additive contributions of the adsorption guest-host interactions and the host-host interactions treated within the framework of linear elasticity into the guest-host free energy allows one to describe in a unified manner the mechanically and adsorption driven deformation of SPCs. The adsorption stress exerted by the guest molecules adsorbed inside the pores causes the same framework deformation as the externally applied pressure of the same magnitude. The threshold stress ansatz implies that the lp-np structural transitions take place abruptly upon achieving the limit of 
elastic stability of the respective phase, similarly to the transition from elastic to plastic deformation. Noteworthy, while the volume changes between the lp and $\mathbf{n p}$ phases of MIL-53 are quite substantial ( $40 \%$ ), the elastic deformations are limited to $2.7 \%$ (for $\mathbf{l p )}$ and $3.6 \%$ (for np). The coherent quantitative analysis of the lp-np transitions in MIL-53 (Cr) based on three types of measurements, mercury porosimetry, adsorption isotherms, and in situ XRD, confirms the validity of the proposed theory.

The proposed thermodynamic treatment of adsorption deformation is not limited to ordered SPCs considered here and can be adopted to any compliant porous solids of different origin, ranging from zeolites ${ }^{32}$ and mesoporous adsorbents ${ }^{36}$ to coal seams in the process of carbon dioxide sequestration at geological conditions ${ }^{37}$.

Acknowledgment. A.V.N. acknowledges the ARO grant W911NF-09-1-0242 and the Région Île-de-France for the support via a Blaise Pascal International Research Chair, administered by the Fondation de l’École normale supérieure.

Supporting Information Available: Representation of the MIL-53 lp and np structures, fits used to determine the bulk moduli of the two phases, and detailed description of the geometrical model used for interpretation of in situ X-ray diffraction data. This material is available free of charge via the Internet at http://pubs.acs.org. 

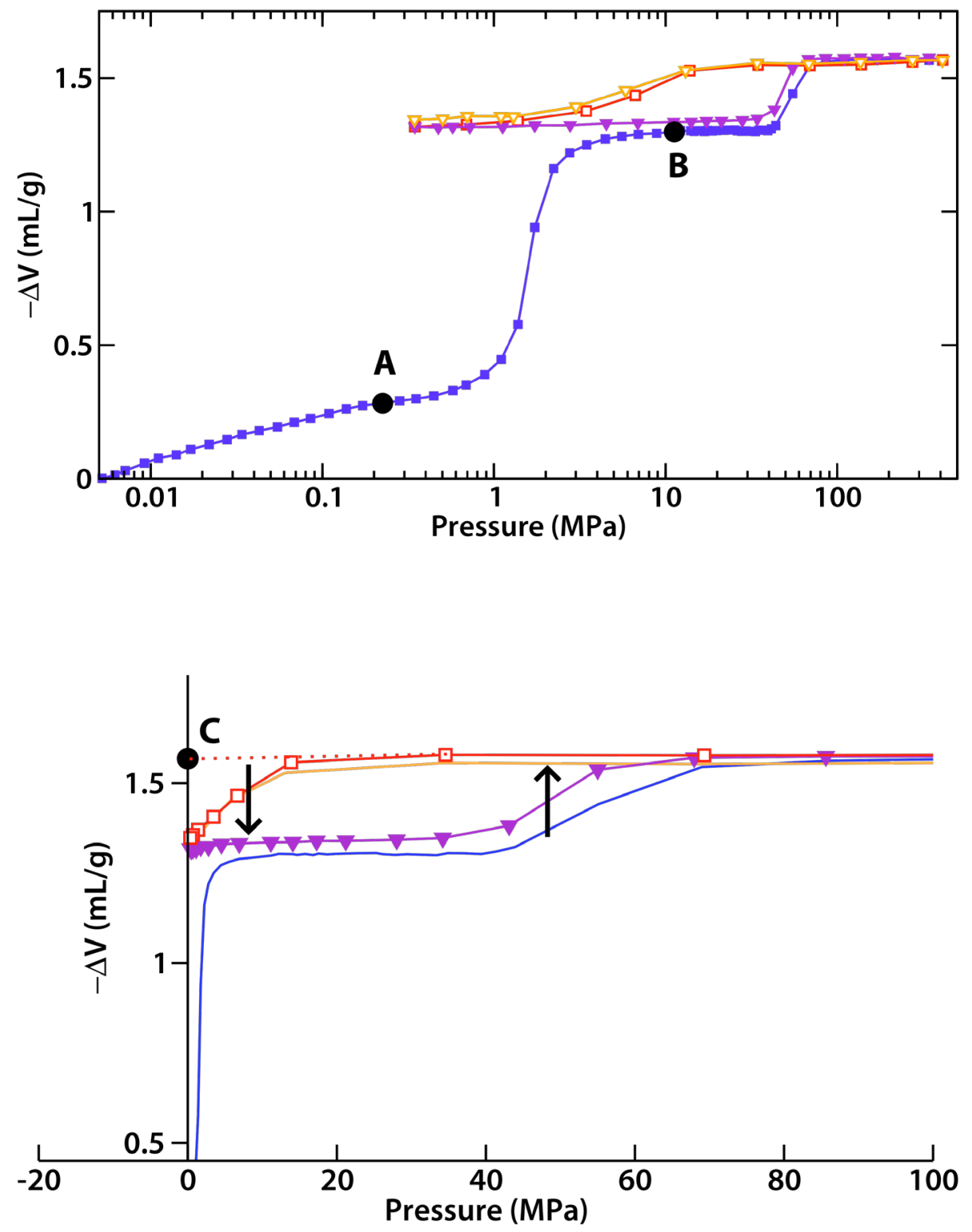

Figure 1. Intrusion-extrusion experiments, featuring the volume variation of the sample $(\Delta V)$ as a function of mechanical pressure. Upper panel: logarithmic pressure scale; lower panel: linear pressure scale. Filled symbols for adsorption, empty symbols for desorption; squares for the first cycle, triangles for the second one. Remarkable points are: A, the end of powder compaction upon compression; $\mathrm{B}$, full interparticle filling upon compression; $\mathrm{C}$, the hypothetical reference state of the np phase, corresponding to $P=0$. 


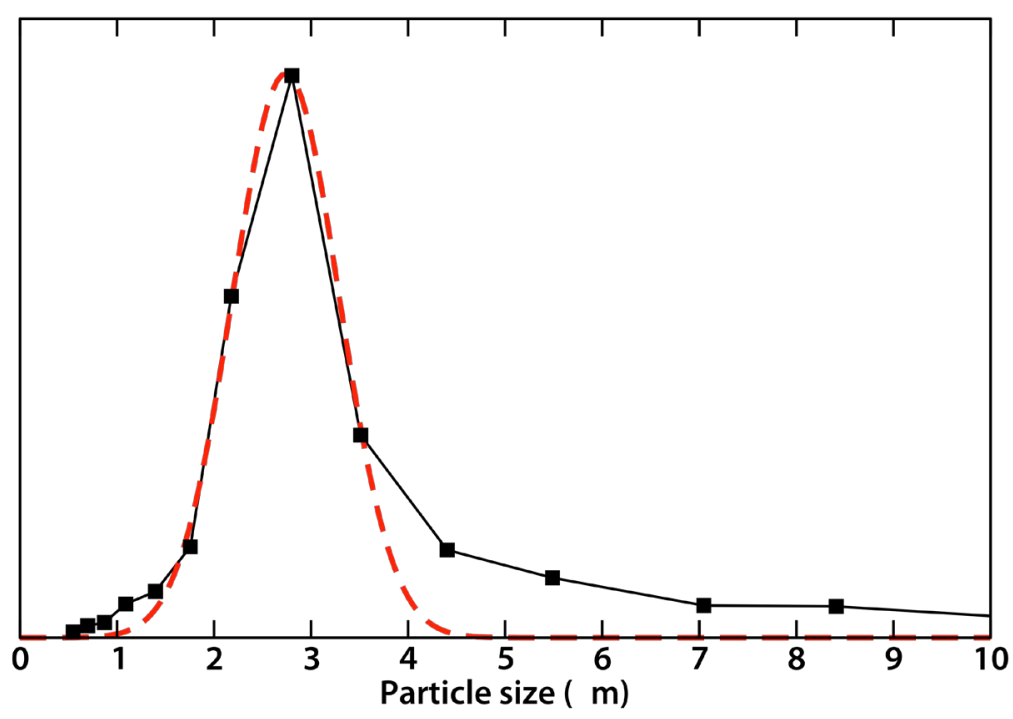

Figure 2. Particle size distribution (in black) and Gaussian fit (in red).
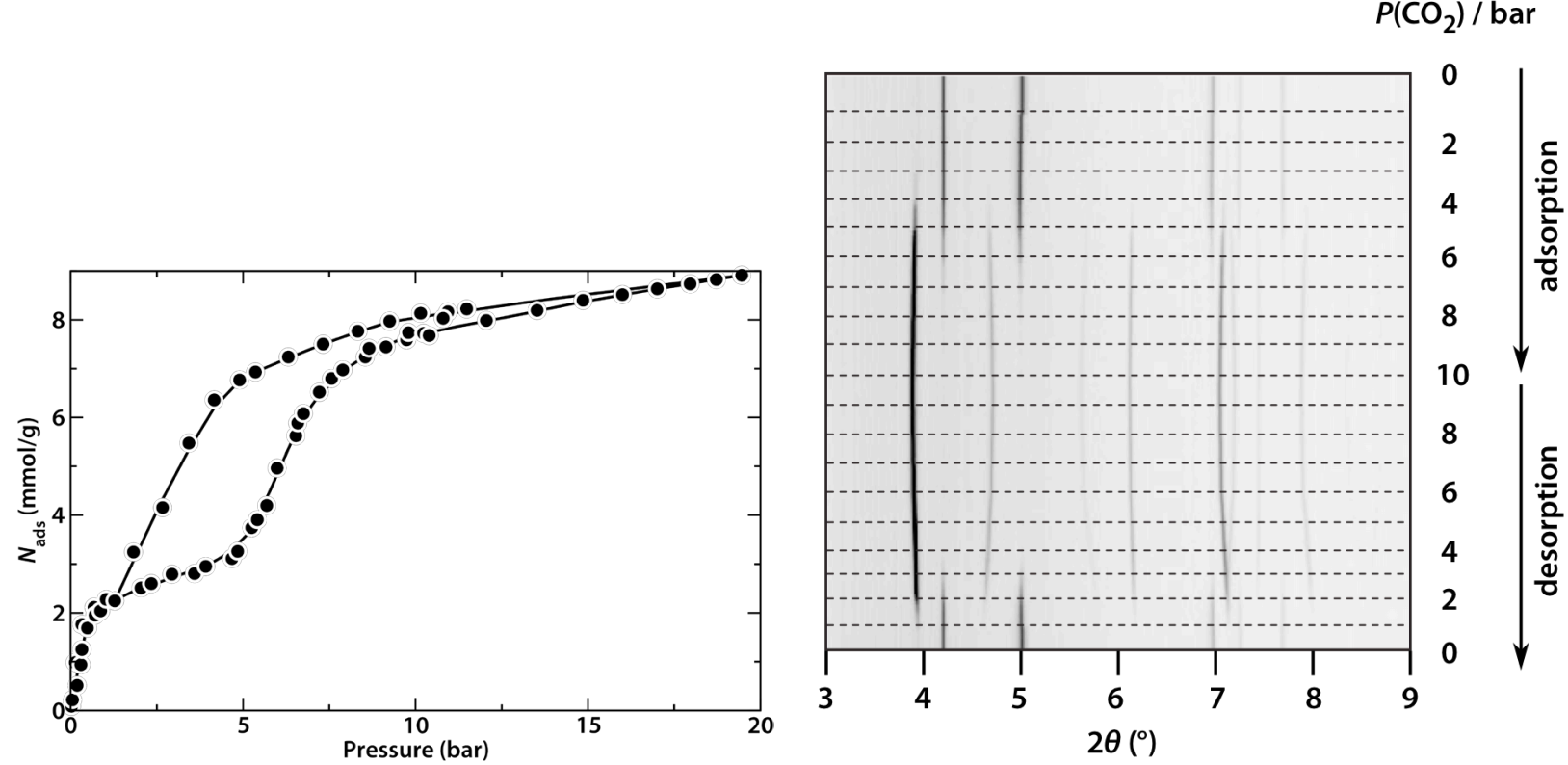

Figure 3: $\mathrm{CO}_{2}$ adsorption and desorption isotherms at $304 \mathrm{~K}$ (left) and structural transformations upon adsorption followed by in situ XRD at $293 \mathrm{~K}$ (right). Figures are redrawn from Ref. 10 (Copyright Wiley-VCH Verlag GmbH \& Co. KGaA. Reproduced with permission.) 


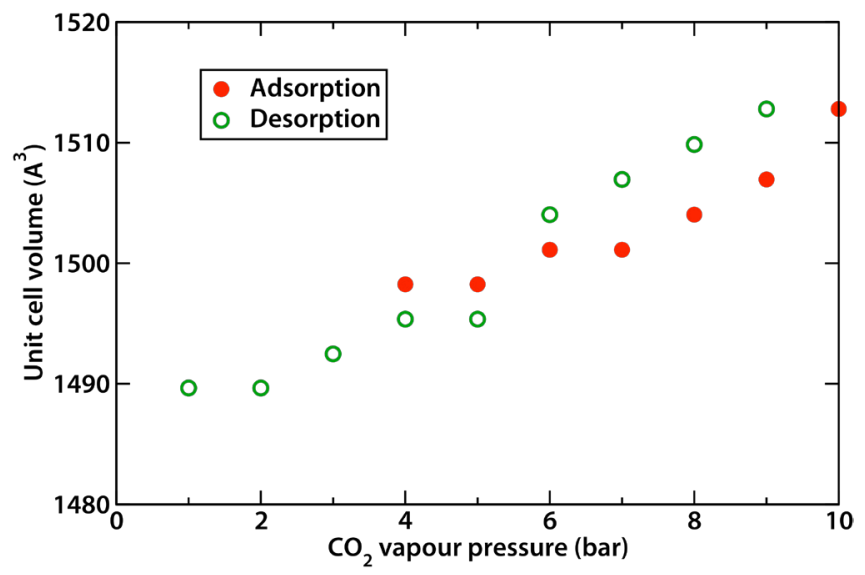

Figure 4: Unit cell volume variation for the lp phase upon $\mathrm{CO}_{2}$ adsorption and desorption at $293 \mathrm{~K}$.

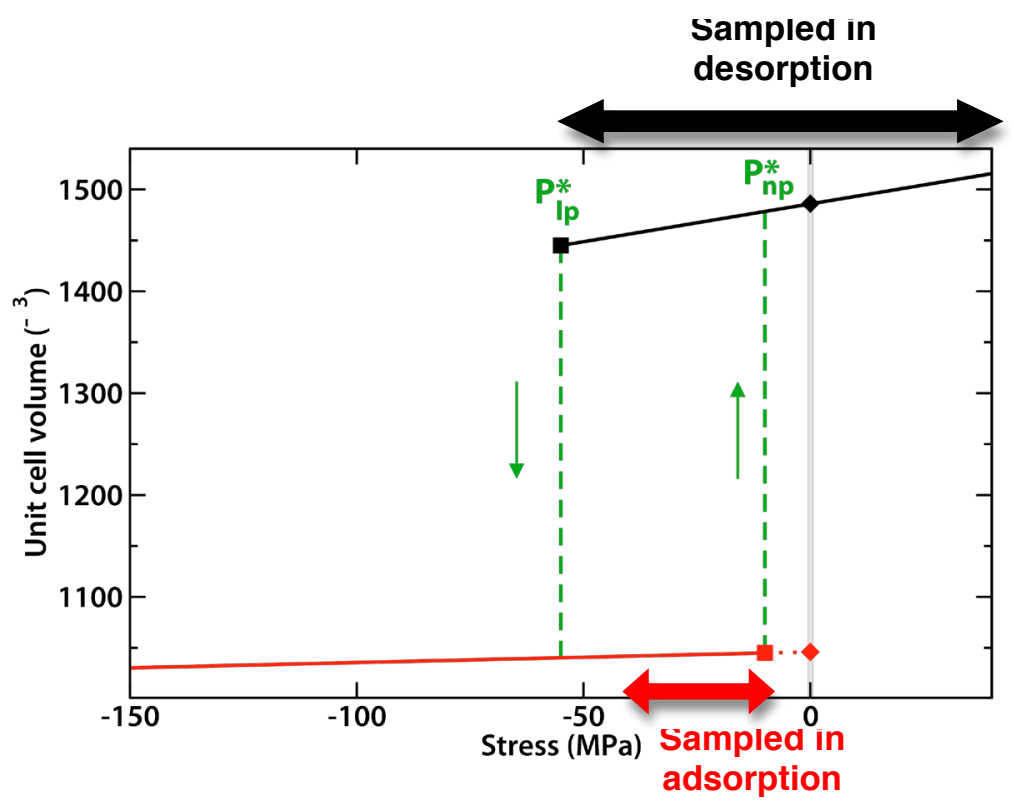

Figure 5: Variation of the unit cell volume in the elastic regime, including threshold pressures and transitions, for both $\mathbf{l p}$ and $\mathbf{n p}$ phases. The region of the stress-strain curves sampled during adsorption are also indicated. 


\begin{tabular}{|c|c|c|}
\hline & Ip phase & np phase \\
\hline$V_{0}$ & $1486 \AA^{3} /$ u.c. & $1046 \AA^{3} /$ u.c. \\
\hline$P^{*}$ & $55( \pm 15) \mathrm{MPa}$ & $10( \pm 10) \mathrm{MPa}$ \\
\hline$K$ & $2 \mathrm{GPa}$ & $10 \mathrm{GPa}$ \\
\hline$\Delta V / V_{0}$ & $2.7 \%$ & $3.6 \%$ \\
\hline
\end{tabular}

Table 1. Mechanical parameters for the two phases of MIL-53 (Cr): reference state volume, $V_{0}$, as determined from crystallography; threshold stress $\left(P^{*}\right)$ and bulk modulus $(K)$ extracted from mercury intrusion/extrusion; relative deformation $\Delta V / V 0$ during the mercury intrusion experiment, ${ }^{22}$ calculated as $\Delta P / K$ where $\Delta P$ is the pressure range of existence of each phase. 


\begin{tabular}{|c|c|c|c|c|}
\hline Host structure & $K_{\mathrm{H}}$ & $N_{0}$ & $\mathrm{~d} K_{\mathrm{H}} / \mathrm{d} V_{\mathrm{c}}$ & $\mathrm{d} N_{0} / \mathrm{d} V_{\mathrm{c}}$ \\
\hline $\mathbf{l p}$ & $2.7 \mathrm{bar}^{-1}$ & 9.3 & $-5.510^{-2} \mathrm{bar}^{-1} \AA^{-3}$ & $0.46 \AA^{-3}$ \\
\hline $\mathbf{n p}$ & $5.2 \mathrm{bar}^{-1}$ & 3.0 & $-1.810^{-2} \mathrm{bar}^{-1} \AA^{-3}$ & $4.910^{-3} \AA^{-3}$ \\
\hline
\end{tabular}

Table 2. Model parameters for adsorption of $\mathrm{CO}_{2}$ on MIL-53(Cr) at $303 \mathrm{~K}$ : Langmuir parameters $\left(K_{\mathrm{H}}\right.$ and $\left.N_{0}\right)$ are taken from fitting the experimental data, ${ }^{10}$ and their derivatives with respect to unit cell volume were optimized to reproduce the threshold stress corresponding to the lp-np and $\mathbf{n p}$-lp transitions in the mercury intrusion measurements (Figure 1) at the characteristic experimental pressures of the lp-np and $\mathbf{n p}-\mathbf{l p}$ transitions in adsorption measurements (Figure 3). Pressure transitions for the first transition, not visible in data from ref. 10, were approximated to 0.2 and 0.3 bar, bracketing the experimental observations by calorimetry. ${ }^{31}$ 


\section{Table of Content Graphic}

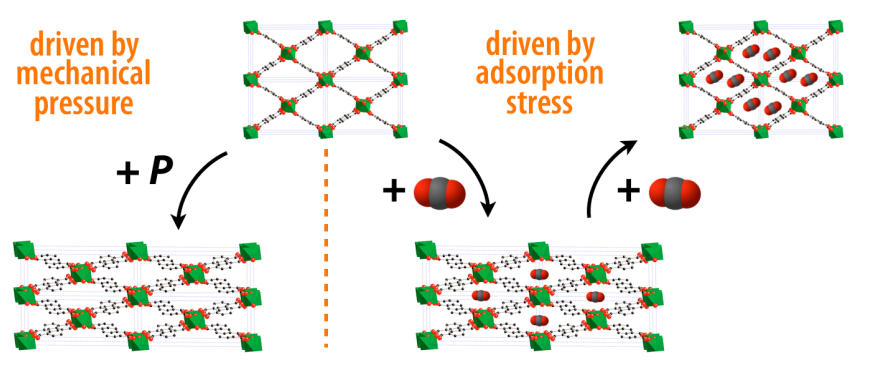




\section{References}

${ }^{1}$ Kitagawa, S.; Uemura, K. Chem. Soc. Rev. 2005, 34, 109-119.

${ }^{2}$ Férey, G.; Serre, C. Chem. Soc. Rev. 2009, 38, 1380-1399.

${ }^{3}$ Kitagawa, S.; Kitaura, R.; Noro, S.-I. Angew. Chem., Int. Ed. 2004, 43, 2334-2375.

${ }^{4}$ Ashleugh, J.; Fletchera, K; Thomas, M.; Rosseinsky, M. J. J. Solid State Chem. 2005, 178, 2491-2510.

${ }^{5}$ Horike, S.; Shimomura, S.; Kitagawa, S. Nature Chem. 2009, 1, 695-704.

${ }^{6}$ Millange, F.; Serre, C.; Férey, G. Chem. Commun. 2002, 822-823.

${ }^{7}$ Loiseau, T.; Serre, C.; Huguenard, C.; Fink, G.; Taulelle, F.; Henry, M.; Bataille, T.; Férey, G. Chem. Eur. J. 2004, 10, 1373-1382.

${ }^{8}$ Bourrelly, S.; Llewellyn, P. L.; Serre, C.; Millange, F.; Loiseau, T.; Férey, G. J. Am. Chem. Soc. 2005, 127, 13519-13521..

${ }^{9}$ Liu, Y.; Her, J.; Dailly, A.; Ramirez-Cuesta, A. J.; Neumann, D. A.; Brown, C. M. J. Am. Chem. Soc. 2008, 130, 11813-11818.

${ }^{10}$ Serre, C.; Bourrelly, S.; Vimont, A.; Ramsahye, N. A.; Maurin, G.; Llewellyn, P. L.; Daturi, M.; Filinchuk, Y.; Leynaud, O.; Barnes, P.; Férey, G. Adv. Mater. 2007, 19, 2246-2251.

${ }^{11}$ Férey, G.; Latroche, M.; Serre, C.; Millange, F.; Loiseau, T.; Percheron-Guegan, A. Chem. Commun. 2003, 2976-2977.

${ }^{12}$ Finsy, V.; Ma, L.; Alaerts, L.; De Vos, D. E.; Baron, G. V.; Denayer, J. F. M. Microporous Mesoporous Mater. 2009, 120, 221-227.

13 Alaerts, L.; Maes, M.; Giebeler, L.; Jacobs, P. A.; Martens, J. A.; Denayer, J. F. M.; Kirschhock, C. E. A.; De Vos, D. E. J. Am. Chem. Soc. 2008, 130, 14170.

${ }^{14}$ Coudert, F.-X.; Mellot-Draznieks, C.; Fuchs, A. H.; Boutin, A. J. Am. Chem. Soc. 2009, 131, 3442-3443.

${ }^{15}$ Coudert, F.-X.; Mellot-Draznieks, C.; Jeffroy, M.; Fuchs, A. H.; Boutin, A. J. Am. Chem. Soc. 2008, 130, 14294-14302.

${ }^{16}$ Coudert, F.-X.; Boutin, A.; Jeffroy, M.; Mellot-Draznieks, C.; Fuchs, A. H. Chem. Phys. Chem. 2011, DOI: $10.1002 /$ cphc. 201000590

${ }^{17}$ Coudert, F.-X.; Mellot-Draznieks, C.; Fuchs, A. H.; Boutin, A. J. Am. Chem. Soc. 2009, 131, 11329-11331.

${ }^{18}$ Coudert, F.-X. Phys. Chem. Chem. Phys. 2010, 12, 10904-10913. 
${ }^{19}$ Boutin, A.; Springuel-Huet, M.-A.; Nossov, A.; Gédéon, A.; Loiseau, T.; Volkringer, C.; Férey, G.; Coudert, F.-X.; Fuchs, A. H. Angew. Chem., Int. Ed. 2009, 48, 8314-8317.

${ }^{20}$ Neimark, A. V.; Coudert, F.-X.; Boutin, A.; Fuchs, A. H. J. Phys. Chem. Lett. 2010, 1, 445449.

${ }^{21}$ Boutin, A.; Coudert, F.-X.; Springuel-Huet, M.-A.; Neimark, A. V.; Férey, G.; Fuchs, A. H. $J$. Phys. Chem. C 2010, 114, 22237-22244.

${ }^{22}$ Beurroies, I.; Boulhout, M.; Llewellyn, P. L.; Kuchta, B; Férey, G.; Serre, C.; Denoyel, R. Angew. Chem., Int. Ed. 2010, 49, 7526-7529.

${ }^{23}$ H. Giesche, Part. Part. Syst. Char., 2006, 23, 9-19.

${ }^{24}$ Mayer, R. P.; Stowe, R. A. J. Colloid Sci. 1965, 20, 893-911.

${ }^{25}$ W. Pabst, E. Gregorova, "Characterization of particles and particle systems", ICT Prague, 2007.

${ }^{26}$ Khan, N. A.; Jhung, S. H. Crys. Growth Des. 2010, 10, 1860-1865.

${ }^{27}$ Zhou, W.; Yildirim, T. Phys. Rev. B 2006, 74, 180301.

${ }^{28}$ Tan, J. C.; Bennet, T. D.; Cheetham, A. K. Proc. Nat. Acad. Sci. USA 2010, 107, 9938-9943.

${ }^{29}$ Chapman, K. W.; Halder, G. J.; Chupas, P. J. J. Am. Chem. Soc. 2008, 130, 10524-10526

${ }^{30}$ Springuel-Huet, M.-A.; Nossov, A.; Adem, Z.; Guenneau, F.; Volkringer, C.; Loiseau, T.; Férey, G.; Gédéon, A. J. Am. Chem. Soc. 2010, 132, 11599-11607.

${ }^{31}$ Coombes, D. S.; Bell, R.; Bourrelly, S.; Llewellyn, P. L.; Mellot- Draznieks, C.; Férey, G. Angew. Chem., Int. Ed. 2008, 47, 8487.

${ }^{32}$ Ravikovitch, P. I.; Neimark, A. V . Langmuir 2006, 22, 10864.

${ }^{33}$ Kowalczyk, P.; Ciach, A.; Neimark, A. V. Langmuir 2008, 24, 6603.

${ }^{34}$ Serre, C.; Mellot-Draznieks, C; Surblé, S.; Audebrand, N.; Filinchuk, Y.; Férey, G. Science 2007, 315, 1828.

${ }^{35}$ Chen, B.; Ma, S.; Zapata, F.; Lobkovsky, E. B.; Yang, J. Inorg. Chem. 2006, 45, 5718-5720.

${ }^{36}$ Gor, G.; Neimark, A.V. Langmuir 2010, 26, 13021.

${ }^{37}$ Yang, K.; Lu, X.; Lin, Y.; Neimark A.V. Energy \& Fuels 2010, 24, 5955. 